\title{
Combination of branched-chain amino acid and angiotensin-converting enzyme inhibitor improves liver fibrosis progression in patients with cirrhosis
}

\author{
HITOSHI YOSHIJI, RYUICHI NOGUCHI, YASUHIDE IKENAKA, KOSUKE KAJI, YOSUKE AIHARA, \\ AKITOSHI DOUHARA, JUNICHI YAMAO, MASAHISA TOYOHARA, AKIRA MITORO, MASAYOSHI SAWAI, \\ MOTOYUKI YOSHIDA, CHIE MORIOKA, MASAO FUJIMOTO, MASAHITO UEMURA and HIROSHI FUKUI
}

The Third Department of Internal Medicine, Nara Medical University, Nara, Japan

Received September 5, 2011; Accepted November 4, 2011

DOI: $10.3892 / \mathrm{mmr} .2011 .676$

\begin{abstract}
An effective therapeutic strategy for suppressing liver fibrosis should improve the overall prognosis of patients with chronic liver diseases. Although enormous efforts are ongoing to develop anti-fibrotic agents, no drugs have yet been approved as anti-fibrotic agents for humans. Insulin resistance (IR) is reportedly involved in the progression of liver fibrosis. The aim of the present study was to evaluate the effect of combination treatment with a clinically used branched-chain amino acid (BCAA) and an angiotensin-converting enzyme inhibitor (ACE-I) on several fibrotic indices in patients with liver cirrhosis under the condition of IR. BCAA granules (Livact; 12 g/day) and/or ACE-I (perindopril; $4 \mathrm{mg} /$ day) were administered, and several indices were analyzed. A 48-month follow-up revealed that the combination treatment with BCAA and ACE-I markedly improved the progression of serum fibrosis markers, whereas single treatment with either BCAA or ACE-I did not exert these inhibitory effects. The plasma level of transforming growth factor- $\beta$ was significantly attenuated almost in parallel with the suppression of serum
\end{abstract}

Correspondence to: Dr Hitoshi Yoshiji, The Third Department of Internal Medicine, Nara Medical University, Shijo-cho 840, Kashihara, Nara 634-8522, Japan

E-mail: yoshijih@naramed-u.ac.jp

Abbreviations: ACE, angiotensin-converting enzyme; ACE-I, angiotensin-converting enzyme inhibitor; Ac-HSCs, activated hepatic stellate cells; AT1-R, angiotensin type-1 receptor; ARB, AT1-R blockers; AT-II, angiotensin-II; BCAA, branched-chain amino acid; CHC, chronic hepatitis C; DM, diabetes mellitus; ECM, extracellular matrix; $\mathrm{HCC}$, hepatocellular carcinoma; $\mathrm{HCV}$, hepatitis $\mathrm{C}$ virus; HOMA, homeostasis model assessment; IR, insulin resistance; NAFLD, non-alcoholic fatty liver disease; RAS, renin-angiotensin system; RFA, radiofrequency ablation; TGF- $\beta$, transforming growth factor $\beta$; VEGF, vascular endothelial growth factor

Key words: branched-chain amino acid, angiotensin-II, liver fibrosis, insulin resistance fibrosis markers. Furthermore, the combined treatment with BCAA and ACE-I improved the serum albumin level and IR, which was evaluated using the homeostasis model assessment method for IR. Taken together, since both BCAA and ACE-I are widely used with safety in clinical practice, these results indicate that this combination therapy may represent a potential new future strategy against liver fibrosis development in patients with liver cirrhosis under the condition of IR.

\section{Introduction}

It is now widely recognized that development of hepatic fibrosis is associated with progression of chronic liver diseases (1-3). Liver fibrosis is characterized by excessive deposition of extracellular matrix (ECM), which leads to severe pathophysiological disturbance in the liver regardless of its etiology, such as hepatitis $\mathrm{C}$ virus (HCV). Advanced fibrotic change was previously considered irreversible, even after withdrawal of the liver-injuring agent. Fibrosis, however, is now regarded as a dynamic and potentially reversible process $(2,4,5)$. Although enormous efforts are ongoing to develop anti-fibrotic agents, no drugs have been approved as anti-fibrotic agents for humans $(4,6)$.

Recent studies have revealed a close relationship between insulin resistance (IR), i.e., co-existence of high blood glucose and insulin levels, and the progression of liver diseases, including liver fibrosis development (7). Cross-sectional human studies have been shown that IR is a consistent finding in patients with type 2 diabetes mellitus (DM) (8). Experimental evidence for the contribution of HCV in the development of IR and DM have been found in a HCV-transgenic mouse model (9). Moreover, several human studies have demonstrated that IR is a risk factor of advanced fibrosis in patients with chronic hepatitis $\mathrm{C}(\mathrm{CHC})$, and IR itself may contribute to the liver fibrosis progression in CHC (10-12). Moreover, a similar close interaction could be observed in patients with other morbidities, such as non-alcoholic fatty liver diseases (NAFLD) (13). Either high glucose or insulin increased the production and expression of collagen genes in activated hepatic stellate cells (HSCs), which play a pivotal role in the liver fibrosis development in vitro $(14,15)$. We previously reported that the IR 
Table I. Demographic characteristics of the enrolled patients.

\begin{tabular}{lcccc}
\hline Characteristics & Control $(\mathrm{G} 1)$ & Combination $(\mathrm{G} 2)$ & ACE-I $(\mathrm{G} 3)$ & BCAA $(\mathrm{G} 4)$ \\
\hline No. of patients & 26 & 28 & 19 & 16 \\
Age (years) & $62.50 \pm 11.50$ & $64.80 \pm 10.60(0.338)$ & $59.40 \pm 12.30(0.390)$ & $63.70 \pm 10.80(0.409)$ \\
Gender (M/F) & $16 / 10$ & $18 / 10(0.888)$ & $12 / 7(0.911)$ & $10 / 6(0.950)$ \\
Etiology (HCV/HBV/other) & $18 / 6 / 2$ & $19 / 8 / 1(0.5)$ & $14 / 4 / 1(0.208)$ & $10 / 5 / 1(0.353)$ \\
Alcohol (<40 g/>40 g/day) & $20 / 6$ & $22 / 6(0.884)$ & $12 / 7(0.314)$ & $12 / 4(0.887)$ \\
T-bil (mg/dl) & $0.80 \pm 0.20$ & $0.70 \pm 0.30$ & $0.80 \pm 0.30$ & $0.90 \pm 0.20$ \\
Alb (g/dl) & $3.38 \pm 0.04$ & $3.41 \pm 0.05$ & $3.35 \pm 0.03$ & $3.33 \pm 0.02$ \\
ALT (IU/l) & $78.80 \pm 31.50$ & $72.30 \pm 40.60(0.099)$ & $74.80 \pm 36.60(0.258)$ & $69.40 \pm 29.60(0.411)$ \\
HOMA-IR & $3.79 \pm 2.92$ & $4.15 \pm 2.76(0.386)$ & $3.28 \pm 3.42(0.247)$ & $3.64 \pm 2.96(0.492)$ \\
FBS (mg/dl) & $113.40 \pm 28.80$ & $106.50 \pm 31.40(0.329)$ & $94.60 \pm 28.80(0.509)$ & $103.80 \pm 35.80(0.191)$ \\
Child-Pugh (A/B) & $21 / 5$ & $22 / 6(0.841)$ & $16 / 3(0.765)$ & $12 / 4(0.658)$ \\
\hline
\end{tabular}

Data represent the means $\pm \mathrm{SD}$. p-values, as compared to the control group, are indicated in parentheses.

itself significantly promoted the liver fibrosis development (16). Accordingly, the agents which induce IR improvement and have anti-fibrotic activity would be useful to improve the development of liver fibrosis under the condition of IR.

The branched-chain amino acids (BCAA) comprise three essential amino acids, namely, leucine, iso-leucine and valine. Previous studies have shown that long-term supplementation with BCAA granules (a mixture of these three amino acids) to patients with liver cirrhosis improved the nutrition status, such as hypoalbuminemia and event-free survival $(17,18)$. In addition, it has been revealed that BCAA suppressed the development of hepatocarcinogenesis in patients with HCV-related cirrhosis, type $2 \mathrm{DM}$ and obesity that is often associated with IR (19). BCAA are also known to modulate insulin signaling. They induce glucose uptake and improve glucose metabolism in rats with liver cirrhosis $(20,21)$. BCAA reportedly improved IR in the animal model as well as in the clinical practice $(22,23)$. A recent study has revealed that BCAA may also exert an anti-fibrotic effect under the condition of IR in murine fibrosis development (22).

It has been reported that the renin-angiotensin system (RAS) is activated in patients with chronic liver diseases, such as cirrhosis (24). It has been revealed that angiotensin-II (AT-II) induced HSC contraction and proliferation (25). We previously reported that a clinically used angiotensin-converting enzyme (ACE) inhibitor (ACE-I) and AT1-R blockers (ARB) significantly attenuated experimental liver fibrosis development and suppressed the activated HSC (Ac-HSC) (26). In addition to BCAA, ACE-I is also known to attenuate the IR status both in vivo and in vitro (27). Since agents which possess both anti-fibrogenic effects and IR-improvement effects would be suitable for patients with IR, this combination regimen of BCAA and ACE-I would be one of the most effective therapeutic modalities.

In the present study, we examined whether the combination of BCAA and ACE-I suppressed the liver fibrosis development in patients with cirrhosis under the condition of IR, and we discussed the possible mechanisms involved.

\section{Patients and methods}

Patients. This study was conducted on 110 patients with cirrhosis associated with hepatocellular carcinoma (HCC), who were admitted to our hospital between May 2004 and July 2006. All patients received curative therapy with percutaneous radiofrequency ablation (RFA) for prior HCC, and were confirmed to be free of any residual HCC by several imaging modalities. The IR index was calculated on the basis of fasting values for glycemia and insulinemia, according to the homeostasis model assessment (HOMA-IR) method, as described previously (28). We also performed the 75-g oral glucose tolerance test (OGTT), and a certified doctor of the Japan Diabetes Society confirmed the status of IR of the enrolled patients. The clinical profiles, laboratory data and characteristics of the patients are shown in Table I. We recommended all patients to stop alcohol intake completely. All patients gave written informed consent before participating in this study. The protocol was approved by the Institutional Review Board (IRB) of the Nara Medical University, and the study was conducted in conformity with the ethical and humane principles. We carefully followed the CONSORT Statement for randomized studies to perform the present analysis.

Study design. After completing the curative therapy for HCC, we enrolled 110 patients. Among them, we were able to follow up 89 patients. These 89 patients were randomly divided into four groups of matching clinical background (e.g., age and gender), IR status and serum fibrosis markers. The control group (Group 1; G1; $\mathrm{n}=26$ ), combination-treated group (Group 2; G2; $\mathrm{n}=28$ ), and the single-treatment groups with either ACE-I (perindopril; $4 \mathrm{mg} / \mathrm{day})(\mathrm{G} 3 ; \mathrm{n}=19)$ or BCAA granules (Livact; $12 \mathrm{~g} /$ day) $(\mathrm{G} 4 ; \mathrm{n}=16)$ were followed up for 48 months. The respective doses of both compounds are standard doses in the clinical practice.

Evaluation of laboratory data. The serum fibrosis markers, namely, hyaluronic acid and type IV collagen 7S (7S-collagen), 

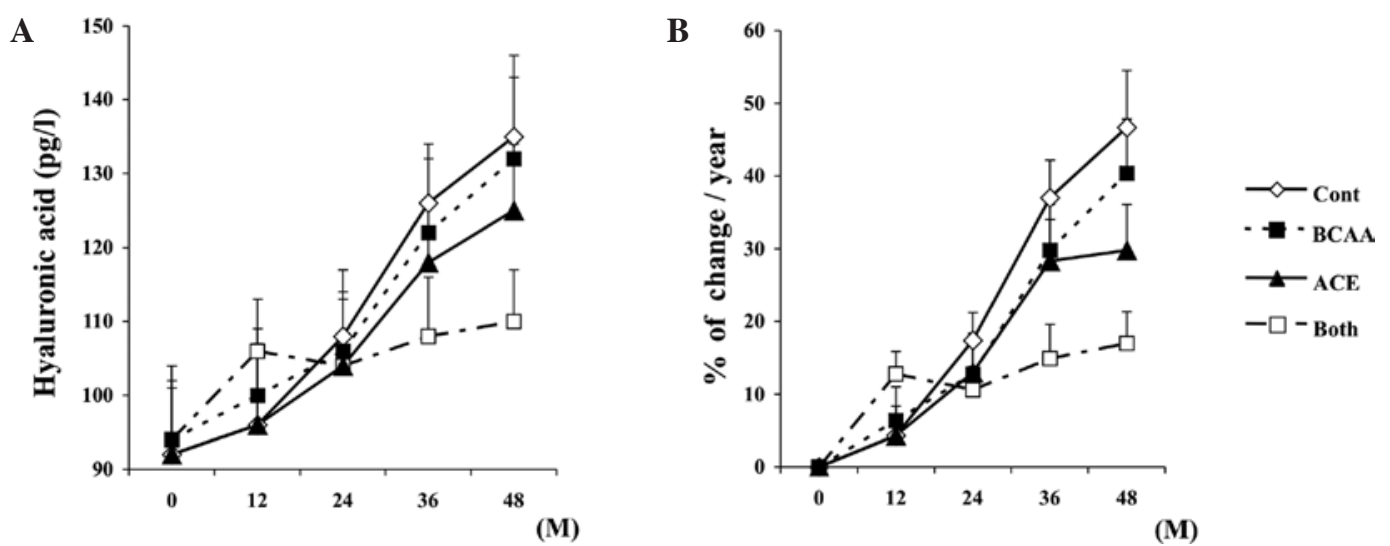

Figure 1. Effects of BCAA and ACE-I on serum hyaluronic acid in patients with cirrhosis under the condition of IR. (A) Hyaluronic acid level during the follow-up (48 months). (B) Percentage of change in the hyaluronic acid level in each group. Cont, untreated control group; ACE, ACE-I-treated group; BCAA, BCAA-treated groups; Both, combination-treated group with ACE-I and BCAA. The data represent the means \pm SD.

A

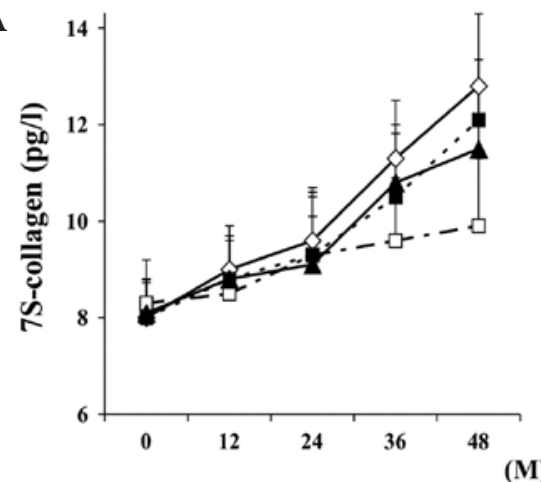

B

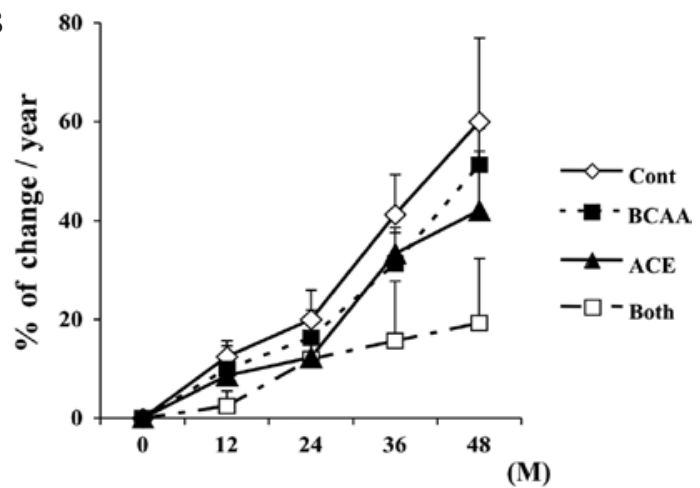

Figure 2. Effects of BCAA and ACE-I on the serum 7S-collagen in patients with cirrhosis under the condition of IR. (A) 7S-collagen level during the follow-up (48 months). (B) Percentage of change in the 7S-collagen level in each group. Cont, untreated control group; ACE, ACE-I-treated group; BCAA, BCAA-treated group; Both, combination-treated group with ACE-I and BCAA. The data represent the means \pm SD.

were measured before and after the treatment in all patients by latex agglutination and enzyme immunoassay (EIA), respectively, by routine laboratory methods. The serum level of transforming growth factor $\beta$ (TGF- $\beta$ ) was measured by ELISA kit (R\&D Systems, Tokyo, Japan) according to the manufacturer's instructions as described previously (29). Other serum biochemical markers, such as alanine transaminase (ALT), were measured by the routine laboratory methods.

Statistical analysis. To assess the statistical significance of the inter-group differences in the quantitative data, Bonferroni's multiple comparison test was performed after one-way analysis of variance (ANOVA). This was followed by Barlett's test to determine the homology of variance.

\section{Results}

Patient characteristics. The characteristics of the enrolled patients are shown in Table I. There were no significant differences among the patients of all groups in terms of age, gender, etiology of the disease or background liver function (Child-Pugh score). Both ACE-I and BCAA are widely used without serious adverse effects, and the safety of their longterm administration has been proven in clinical practice. In the present study, there were no severe toxic effects in any group, and no abnormal laboratory data were found that could likely be related to treatment with either ACE-I or BCAA. Accordingly, after randomization, we were able to follow up all of the enrolled patients in each group until the end of the study protocol.

Serum fibrosis markers. To examine the therapeutic effects of BCAA and/or ACE-I on the progression of liver fibrosis in the patients with cirrhosis, we compared several fibrosis markers for 48 months after the respective treatment. As shown in Fig. 1A, there were differences in the serum hyaluronic acid level among the groups. Without treatment (G1), the mean serum hyaluronic acid level increased during the follow-up (48 months). The combination treatment with BCAA and ACE-I (G2) significantly suppressed the increase in serum hyaluronic acid level as compared to the control group (G1) for 48 months. On the other hand, single treatment with either ACE-I (G3) or BCAA (G4) did not exert any inhibitory effect as compared to the control group (G1). The percentage of change in the hyaluronic acid among the groups showed results similar to those of the definite mean serum hyaluronic acid level (Fig. 1B). Similar results were observed in the 7S-collagen levels (Fig. 2). 
Table II. Changes of several markers in the enrolled patients following 12-month treatment.

\begin{tabular}{|c|c|c|c|c|c|c|c|c|}
\hline & \multicolumn{2}{|c|}{ Control (G1) } & \multicolumn{2}{|c|}{ Combination (G2) } & \multicolumn{2}{|c|}{ ACE-I (G3) } & \multicolumn{2}{|c|}{ BCAA (G4) } \\
\hline & Before & After & Before & After & Before & After & Before & After \\
\hline Mean BP (mmHg) & $138 \pm 21^{\mathrm{a}}$ & $132 \pm 18$ & $140 \pm 36$ & $120 \pm 25^{b}$ & $138 \pm 29$ & $120 \pm 17^{b}$ & $143 \pm 16$ & $136 \pm 18$ \\
\hline Alb (g/dl) & $3.40 \pm 0.04$ & $3.40 \pm 0.05$ & $3.40 \pm 0.05$ & $3.50 \pm 0.04$ & $3.40 \pm 0.03$ & $3.40 \pm 0.04$ & $3.30 \pm 0.02$ & $3.50 \pm 0.05$ \\
\hline ALT (IU/l) & $78.80 \pm 31.50$ & $72.60 \pm 30.30$ & $72.30 \pm 40.60$ & $65.80 \pm 34.40$ & $74.80 \pm 36.65$ & $70.20 \pm 28.60$ & $69.40 \pm 29.60$ & $68.80 \pm 31.30$ \\
\hline FBS (mg/dl) & $113.40 \pm 28.80$ & $107.80 \pm 31.20$ & $106.50 \pm 31.40$ & $94.60 \pm 28.80$ & $99.80 \pm 27.60$ & $92.70 \pm 28.90$ & $103.80 \pm 35.80$ & $95.40 \pm 31.10$ \\
\hline HOMA-R & $3.79 \pm 2.92$ & $3.61 \pm 2.88$ & $4.15 \pm 2.76$ & $2.82 \pm 1.94^{\mathrm{b}}$ & $3.88 \pm 2.79$ & $3.14 \pm 2.27$ & $3.55 \pm 3.01$ & $2.75 \pm 2.08$ \\
\hline
\end{tabular}

${ }^{a}$ Data represent the means $\pm \mathrm{SD} .{ }^{\mathrm{b}, \mathrm{c}}$ Statistically significant as compared to before treatment $(\mathrm{p}<0.05$ and $\mathrm{p}<0.01$, respectively).

A

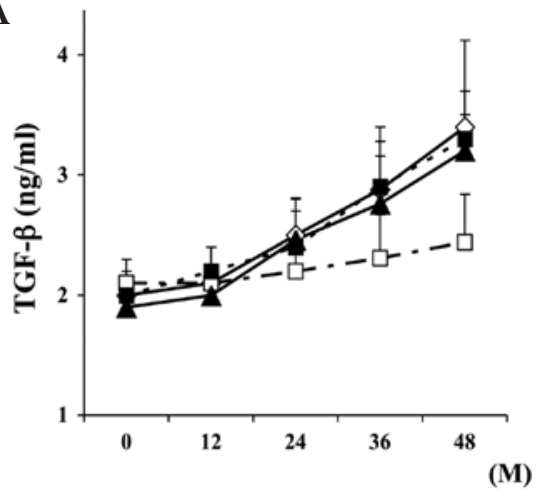

B

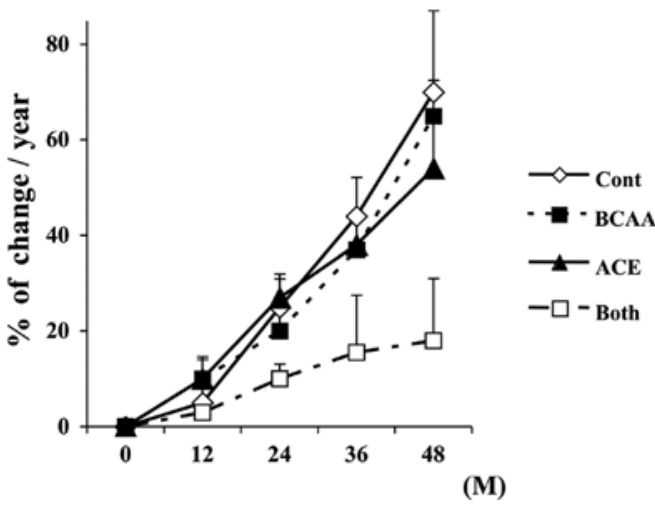

Figure 3. Effects of BCAA and ACE-I on the serum TGF- $\beta$ in the patients with cirrhosis under the condition of IR. (A) TGF- $\beta$ level during the follow-up (48 months). (B) Percentage of change in the TGF- $\beta$ level in each group. Cont, untreated control group; ACE, ACE-I-treated group; BCAA, BCAA-treated groups; Both, combination-treated group with ACE-I and BCAA. The data represent the means \pm SD.
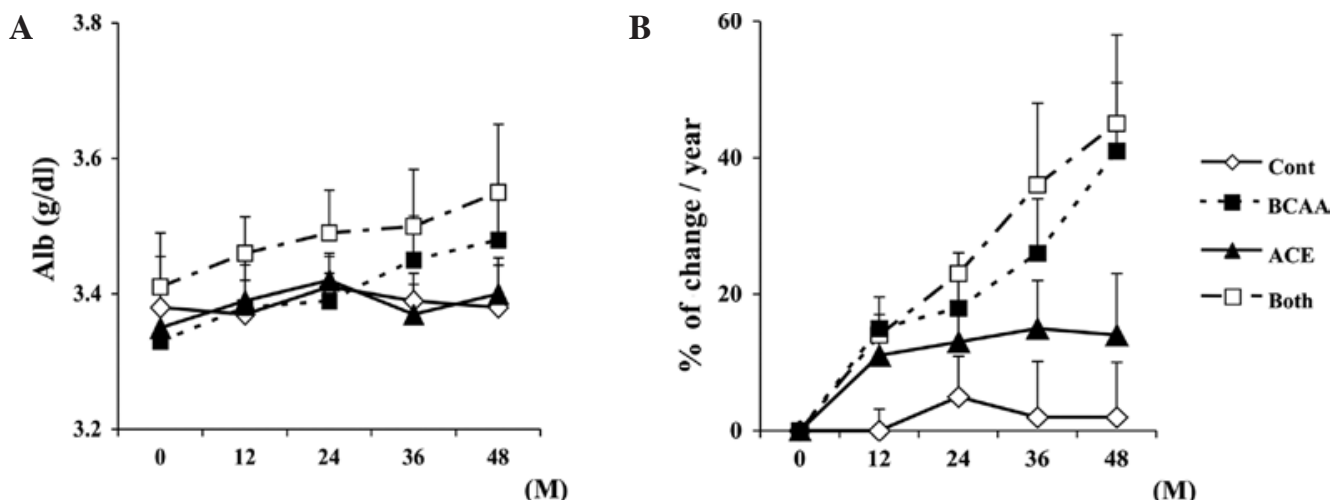

Figure 4. Effects of BCAA and ACE-I on the serum albumin in the patients with cirrhosis under the condition of IR. (A) Albumin level during the follow-up (48 months). (B) Percentage of change in the albumin level in each group. Cont, untreated control group; ACE, ACE-I-treated group; BCAA, BCAA-treated groups; Both, combination-treated group with ACE-I and BCAA. The data represent the means \pm SD.

Biochemical markers and serum TGF- $\beta$ level. We compared several markers among the enrolled patients following the 12-month treatment (Table II). The mean blood pressure decreased in the combination- and ACE-I-treated groups, but there were no significant differences between the groups. Furthermore, there were no significant differences in the ALT level between the combination-treated and other groups, indicating that the suppressive effect of this combination treatment on fibrosis progression was not due to anti-hypertensive or cytoprotective activities. Since TGF- $\beta$ is known as a key cytokine in liver fibrosis development, we next measured the TGF- $\beta$ level in all groups. The alterations of the TGF- $\beta$ level were almost parallel to those of the fibrosis markers. The BCAA + ACE-I treatment significantly attenuated the TGF- $\beta$ level at the end of the study (Fig. 3). As shown in Fig. 4, on the other hand, the serum albumin level significantly increased in the BCAA + ACE-I-treated group. In addition to the combination-treated group, single treatment with BCAA also improved the albumin level, which is consistent with a previous report (19). We also examined the alteration of the 
IR status by the HOMA-IR score. As shown in Table II, the combination treatment with BCAA and ACE-I for 12 months significantly decreased the median HOMA-IR score. Single treatment with BCAA or ACE-I also tended to decrease the HOMA-IR level, but the decrease was not significant.

\section{Discussion}

In the present study, we found that treatment with the clinically used BCAA and ACE-I markedly inhibited fibrosis progression in patients with cirrhosis under the condition of IR, along with suppression of several indices. In human livers, fibrogenesis underlies the development of HCC in at least $90 \%$ of cases, and HCC is an ominous complication of cirrhosis in $30 \%$ of patients (30). The risk of HCC reportedly increases in parallel with the progression of hepatic fibrosis (31). Furthermore, the existence of fibrosis itself accelerates experimental hepatocarcinogenesis (32). Taken together, an effective therapeutic strategy for suppressing liver fibrosis should improve the overall prognosis of patients with chronic liver diseases. We recently observed that the combination treatment with BCAA and ACE-I markedly inhibited cumulative HCC recurrence after curative therapy under the condition of IR (33). Analysis of liver fibrosis development in the same clinical groups revealed that this combined regimen markedly inhibited fibrosis progression along with suppression of several indices. These results indicated that the combination treatment with BCAA and ACE-I would improve the prognosis in patients with liver cirrhosis under the condition of IR.

In this study, we observed that the anti-fibrotic effect of this combination treatment was parallel to suppression of the TGF- $\beta$ level. It is well known that TGF- $\beta$ is a central key cytokine in liver fibrosis development (4). We previously reported that ACE-I, even at a clinically comparable low dose, significantly attenuated experimental liver fibrosis development along with suppression of TGF- $\beta(26,34)$. In clinical practice, a retrospective study on liver transplanted to patients with $\mathrm{CHC}$, re-infection with $\mathrm{HCV}$ revealed that the patients receiving ACE-I had significantly less fibrosis progression than those receiving other types of drugs (35). In the present study, we found that the combination treatment with ACE-I and BCAA significantly suppressed several serum fibrosis markers along with TGF- $\beta$ inhibition as compared to either ACE-I or BCAA alone. The combination treatment with different agents was shown to exert more potent anti-fibrotic effect as compared to either single agent (36). It is well known that Ac-HSCs play a pivotal role in liver fibrogenesis, and that Ac-HSCs are one of the main cells producing TGF- $\beta$ and collagen (4). The exact mechanism of the inhibitory effect in this study remains obscure at this time. However, we observed that serum TGF- $\beta$ and $7 \mathrm{~S}$-collagen were suppressed almost in parallel by this combination regimen, whereas the ALT status was not altered. These results indicated that the anti-fibrotic effect of this combination regimen was, at least partly, mediated through inhibition of Ac-HSC.

In addition, several different mechanisms are likely to be involved in the suppressive effect on the liver fibrosis progression. Angiogenesis is known to play an important role in many biological phenomena (37). Although previous studies conducted to determine the molecular processes associated with fibrosis and angiogenesis were performed independently, recent studies have revealed that both biological phenomena emerged synergistically (38). We and other groups reported that neovascularization significantly increased during liver fibrosis development (38-40). We previously reported that ACE-I and BCAA exerted an anti-angiogenic activity (41). The combination treatment of BCAA and ACE-I in patients with liver cirrhosis under the condition of IR significantly decreased several angiogenic indices, such as the serum level of vascular endothelial growth factor (VEGF) (33). It may be possible that the anti-fibrotic effect of this combination treatment is, to some extent, mediated by suppression of neovascularization in the liver. Also, the IR status directly accelerates the liver fibrosis development via stimulation of Ac-HSC and neovascularization (16). We observed that IR was significantly improved by the combination treatment, indicating that this effect is also likely to play an important role in the clinical beneficial effect. These coordinating different activities should contribute to the inhibitory effect of this combination regimen. Further studies are required to elucidate the exact role of each activity in the future.

In conclusion, we demonstrated that the combination treatment with clinically used safe agents, namely, BCAA and ACE-I, markedly inhibited fibrosis progression in patients with cirrhosis under the condition of IR along with suppression of several indices, such as TGF- $\beta$ and HOMA-IR. Although a large-scale long-term follow-up case-control study is required, this combined treatment may represent a potential new strategy for suppression of liver fibrosis.

\section{References}

1. Friedman SL: Mechanisms of hepatic fibrogenesis. Gastroenterology 134: 1655-1669, 2008.

2. Fallowfield $\mathbf{J}$ and Hayes P: Pathogenesis and treatment of hepatic fibrosis: is cirrhosis reversible? Clin Med 11: 179-183, 2011.

3. Fallowfield JA: Therapeutic targets in liver fibrosis. Am J Physiol Gastrointest Liver Physiol 300: G709-G715, 2011.

4. Friedman SL: Liver fibrosis - from bench to bedside. J Hepatol 38 (Suppl 1): 38-53, 2003.

5. Povero D, Busletta C, Novo E, et al: Liver fibrosis: a dynamic and potentially reversible process. Histol Histopathol 25: 1075-1091, 2010.

6. Poelstra K and Schuppan D: Targeted therapy of liver fibrosis/ cirrhosis and its complications. J Hepatol 55: 726-728, 2011.

7. Yoshiji H, Noguchi R, Ikenaka Y, Kaji K, Aihara Y and Fukui H: Impact of renin-angiotensin system in hepatocellular carcinoma. Curr Cancer Drug Targets 11: 431-441, 2011.

8. Kahn CR: Insulin resistance, insulin insensitivity, and insulin unresponsiveness: a necessary distinction. Metabolism 27: 1893-1902, 1978.

9. Shintani Y, Fujie H, Miyoshi $\mathrm{H}$, et al: Hepatitis $\mathrm{C}$ virus infection and diabetes: direct involvement of the virus in the development of insulin resistance. Gastroenterology 126: 840-848, 2004.

10. Hui JM, Sud A, Farrell GC, et al: Insulin resistance is associated with chronic hepatitis $\mathrm{C}$ virus infection and fibrosis progression [corrected]. Gastroenterology 125: 1695-1704, 2003.

11. Petrides AS, Vogt C, Schulze-Berge D, Matthews D and Strohmeyer G: Pathogenesis of glucose intolerance and diabetes mellitus in cirrhosis. Hepatology 19: 616-627, 1994.

12. Petit JM, Bour JB, Galland-Jos C, et al: Risk factors for diabetes mellitus and early insulin resistance in chronic hepatitis C. J Hepatol 35: 279-283, 2001.

13. Bugianesi E, McCullough AJ and Marchesini G: Insulin resistance: a metabolic pathway to chronic liver disease. Hepatology 42: 987-1000, 2005.

14. Sugimoto R, Enjoji M, Kohjima M, et al: High glucose stimulates hepatic stellate cells to proliferate and to produce collagen through free radical production and activation of mitogenactivated protein kinase. Liver Int 25: 1018-1026, 2005. 
15. Svegliati-Baroni G, Ridolfi F, Di Sario A, et al: Insulin and insulin-like growth factor-1 stimulate proliferation and type I collagen accumulation by human hepatic stellate cells: differential effects on signal transduction pathways. Hepatology 29: 1743-1751, 1999.

16. Kaji K, Yoshiji H, Kitade M, et al: Impact of insulin resistance on the progression of chronic liver diseases. Int J Mol Med 22: 801-808, 2008

17. Marchesini G, Bianchi G, Merli M, et al: Nutritional supplementation with branched-chain amino acids in advanced cirrhosis: a double-blind, randomized trial. Gastroenterology 124: 1792-1801, 2003.

18. Muto Y, Sato S, Watanabe A, et al: Effects of oral branchedchain amino acid granules on event-free survival in patients with liver cirrhosis. Clin Gastroenterol Hepatol 3: 705-713, 2005.

19. Muto Y, Sato S, Watanabe A, et al: Overweight and obesity increase the risk for liver cancer in patients with liver cirrhosis and long-term oral supplementation with branched-chain amino acid granules inhibits liver carcinogenesis in heavier patients with liver cirrhosis. Hepatol Res 35: 204-214, 2006.

20. Nishitani S, Ijichi C, Takehana K, Fujitani S and Sonaka I: Pharmacological activities of branched-chain amino acids: specificity of tissue and signal transduction. Biochem Biophys Res Commun 313: 387-389, 2004.

21. Nishitani S, Takehana K, Fujitani S and Sonaka I: Branchedchain amino acids improve glucose metabolism in rats with liver cirrhosis. Am J Physiol Gastrointest Liver Physiol 288 : G1292-G1300, 2005.

22. Iwasa J, Shimizu M, Shiraki M, et al: Dietary supplementation with branched-chain amino acids suppresses diethylnitrosamineinduced liver tumorigenesis in obese and diabetic C57BL/ KsJ-db/db mice. Cancer Sci 101: 460-467, 2010.

23. Kawaguchi T, Nagao Y, Matsuoka H, Ide T and Sata M: Branched-chain amino acid-enriched supplementation improves insulin resistance in patients with chronic liver disease. Int J Mol Med 22: 105-112, 2008

24. Helmy A, Jalan R, Newby DE, Hayes PC and Webb DJ: Role of angiotensin II in regulation of basal and sympathetically stimulated vascular tone in early and advanced cirrhosis. Gastroenterology 118: 565-572, 2000.

25. Bataller R, Gines P, Nicolas JM, et al: Angiotensin II induces contraction and proliferation of human hepatic stellate cells. Gastroenterology 118: 1149-1156, 2000

26. Yoshiji H, Kuriyama S, Yoshii J, et al: Angiotensin-II type 1 receptor interaction is a major regulator for liver fibrosis development in rats. Hepatology 34: 745-750, 2001

27. Yoshiji H, Kuriyama S and Fukui H: Angiotensin-I-converting enzyme inhibitors may be an alternative anti-angiogenic strategy in the treatment of liver fibrosis and hepatocellular carcinoma. Possible role of vascular endothelial growth factor. Tumour Biol 23: 348-356, 2002.
28. Fartoux L, Poujol-Robert A, Guechot J, Wendum D, Poupon R and Serfaty L: Insulin resistance is a cause of steatosis and fibrosis progression in chronic hepatitis C. Gut 54: 1003-1008, 2005.

29. Yoshiji H, Kuriyama S, Noguchi R, et al: Combination of interferon-beta and angiotensin-converting enzyme inhibitor, perindopril, attenuates the murine liver fibrosis development. Liver Int 25: 153-161, 2005.

30. Befeler AS and Di Bisceglie AM: Hepatocellular carcinoma: diagnosis and treatment. Gastroenterology 122: 1609-1619, 2002.

31. Okita K, Sakaida I and Hino K: Current strategies for chemoprevention of hepatocellular carcinoma. Oncology 62 (Suppl 1): 24-28, 2002.

32. Sakaida I, Hironaka K, Uchida K, Suzuki C, Kayano K and Okita K: Fibrosis accelerates the development of enzyme-altered lesions in the rat liver. Hepatology 28: 1247-1252, 1998.

33. Yoshiji H, Noguchi R, Ikenaka Y, et al: Combination of branched-chain amino acids and angiotensin-converting enzyme inhibitor suppresses the cummulative recurrence of hepatocellular carcinoma: A randomized control trail. Oncol Rep 26 1547-1553, 2011

34. Yoshiji H, Yoshii J, Ikenaka Y, et al: Suppression of the reninangiotensin system attenuates vascular endothelial growth factor-mediated tumor development and angiogenesis in murine hepatocellular carcinoma cells. Int J Oncol 20: 1227-1231, 2002.

35. Rimola A, Londono MC, Guevara G, et al: Beneficial effect of angiotensin-blocking agents on graft fibrosis in hepatitis $\mathrm{C}$ recurrence after liver transplantation. Transplantation 78: 686-691, 2004.

36. Yoshiji H, Noguchi R, Ikenaka Y, et al: Cocktail therapy with a combination of interferon, ribavirin and angiotensin-II type 1 receptor blocker attenuates murine liver fibrosis development. Int J Mol Med 28: 81-88, 2011.

37. Carmeliet P: Angiogenesis in health and disease. Nat Med 9: 653-660, 2003.

38. Kalluri R and Sukhatme VP: Fibrosis and angiogenesis. Curr Opin Nephrol Hypertens 9: 413-418, 2000.

39. Yoshiji H, Kuriyama S, Yoshii J, et al: Vascular endothelial grow th factor and receptor interaction is a prerequisite for murine hepatic fibrogenesis. Gut 52: 1347-1354, 2003.

40. Ankoma-Sey V, Wang Y and Dai Z: Hypoxic stimulation of vascular endothelial growth factor expression in activated rat hepatic stellate cells. Hepatology 31: 141-148, 2000.

41. Yoshiji H, Noguchi R, Kaji K, et al: Attenuation of insulinresistance-based hepatocarcinogenesis and angiogenesis by combined treatment with branched-chain amino acids and angiotensin-converting enzyme inhibitor in obese diabetic rats. J Gastroenterol 45: 443-450, 2010. 